\title{
Specificity of inhibitory deficits in normal aging and Alzheimer's disease
}

Fabienne Collette ${ }^{1,2,3, *}$, Christina Schmidt ${ }^{1,2,3}$, Christine Scherrer ${ }^{1}$,

Stéphane Adam ${ }^{1}$, Eric Salmon ${ }^{2,4}$

${ }^{1}$ Cognitive Sciences Department, University of Liège, Liège, Belgium

${ }^{2}$ Cyclotron Research Center, University of Liège, Liège, Belgium

${ }^{3}$ Fonds National de la Recherche Scientifique, Belgium

${ }^{4}$ Neurology Department, CHU, Liège, Belgium

Running head title: Inhibition in normal aging and $\mathrm{AD}$

* Correspondence concerning this article should be addressed to Fabienne Collette, Neuropsychology Unit, University of Liège, Boulevard du Rectorat 3 (B33), 4000 Liège, Belgium.

Telephone: (32) 43662274

Fax: (32) 43662875

E-mail: f.collette@ulg.ac.be 


\begin{abstract}
Deficits of suppression abilities are frequently observed in normal aging and Alzheimer's disease. However, few studies have explored these deficits in the two populations simultaneously using a large battery of tasks. The aim of the present study was to explore if the pattern of performance presented by elderly subjects and AD patients is in agreement with theoretical frameworks [104; see also 81], distinguishing between the concepts of inhibition (a voluntary suppression of irrelevant information) and interference (an automatic suppression process occurring prior to conscious awareness). The results obtained demonstrated that (1) there is an alteration of the inhibitory process in normal elderly subjects; (2) inhibitory and interference resolution processes are quantitately less efficient in $\mathrm{AD}$, since these patients present a correct performance only for information which leaves weak traces in memory.
\end{abstract}

Keywords: inhibition; interference resolution; normal aging; Alzheimer’s disease; executive functions 


\section{Introduction}

Inhibition (or suppression abilities) is a basic aspect of cognitive and emotional functioning, which is involved in the performance of numerous tasks and processes [10,15,22,30,33,81]. Recently, several theoretical frameworks have been proposed to explain the inhibitory effects reported in the literature in various normal and pathological populations. For instance, inhibition was specifically related to working memory by Hasher, Zacks, and May [52,53], who described three general inhibitory functions that operate at different times in the information processing sequence: the access function, preventing access to irrelevant information; the deletion function, suppressing information that either is or becomes irrelevant; and the restraint function, which operates when strong responses are triggered by a familiar cue but do not have to be produced. Other authors viewed inhibition as a general process operating in various cognitive domains. In that context, Dempster and Corkill [34,35] have suggested making a distinction between perceptual, motor and verbal inhibition. Inhibitory tasks were also classified according to the following three dimensions: (1) intentional vs. unintentional, (2) behavioral vs. cognitive, and (3) inhibition vs. interference [46]. More generally, Nigg [81] suggested dissociating effortful inhibitory processes (for example, cognitive inhibition, behavioral inhibition and oculo-motor inhibition) from automatic inhibition of attention (concerning inhibition of irrelevant spatial localizations or of recently inspected stimuli). Finally, Kipp Harnishfeger [45,46,104], on the basis of task-analyses, proposed to distinguish between the concepts of inhibition and interference. In that theoretical framework, inhibitory control corresponds to a voluntary suppression of the information, and interference resolution represents a gating mechanism preventing the processing of distracting information. More precisely, Kipp Harnishfeger proposed that interference resolution consists in an automatic process occurring prior to conscious awareness while inhibition results when a stimulus is classified as irrelevant for the ongoing task and is then consciously suppressed.

It must nevertheless be emphasized that the terms "inhibition" and "interference resolution" were used in slightly different ways by authors interested in suppression processes. For example, the access inhibitory function proposed by Hasher, Zacks, and May [52,53] could be considered as an interference resolution process in the context of the Kipp Harnishfeger proposal [104]. Similarly, 
performance on the Stroop task was considered as reflecting sensitivity to interference although the task requires the voluntary suppression of an irrelevant cognitive process. In this paper, the term "inhibition" will be used to refer to functions that are voluntary and require cognitive control whereas the term "interference resolution" will refer to functions that are more automatic and require no (or less) cognitive control.

Changes in inhibitory / interference resolution abilities have been reported across the entire life span [e.g., 45,46]. During normal aging, larger Stroop effects were observed, indicating difficulties suppressing an overlearned response (e.g., reading) in order to produce a less-practiced response (e.g., naming) $[37,47,57,64,94]$. Similarly, lesser negative priming effects have often been demonstrated in elderly subjects in comparison to young subjects, indicating a less efficient suppression of the irrelevant dimension of the stimulus [51,62,75,95,98]. With regard to semantic processes, difficulties inhibiting the reading and processing of irrelevant information embedded in a text $[26,36,44]$, suppressing no longer relevant information produced following a reading task [49] or restraining the production of a word that is strongly induced by the context of a sentence [6] have frequently been reported. Motor inhibition deficits were also observed on the stop-signal task [66,74], the go/no-go task [80] and the antisaccade task [18]. Finally, decreased directed forgetting abilities in working and episodic memory have also been reported $[9,106]$. However, a negative effect of aging on suppression abilities has not been systematically observed (see [63] for the Stroop task, [27,66,67,96,102] for negative priming and [48] for inhibition of return), or is sometimes explained by more basic cognitive variables, such as a decrease in processing speed [88,89,101], fluid intelligence abilities [93] or circadian preferences [59; see 105].

Inhibitory and interference resolution deficits have also been frequently reported in the first stages of Alzheimer's disease (AD) [for reviews, see 4,11,24]. Typically, the Stroop effects (assessed by response times and response accuracy) are considerably larger in $\mathrm{AD}$ patients in comparison to healthy elderly controls, even after adjustment of suppression scores for processing speed $[5,16,40,65,94]$. Similarly, a less reliable or absent negative priming effect has been observed in these patients [3,96; see, however, 67]. With regard to semantic inhibition, Collette et al. [23] observed a weaker ability to suppress semantically related but task-irrelevant responses on the Hayling task [17], 
and Duchek et al. [36] found that AD patients are disproportionately influenced by semantically related distracting information during a reading task. Finally, perseverations [41,42,91] and intrusion errors $[2,12,19,69]$ are frequently produced by $\mathrm{AD}$ patients during list recall performance, indicating an impairment of the suppression processes associated with explicit memory tasks.

Nevertheless, not all inhibitory /interference resolution processes appear to be impaired by AD. Little evidence of dysfunction has been found in tasks assessing motor response inhibition [3; see, however, 25]. Similarly, tasks requiring suppression processes considered to be more automatic [e.g., 81] also appear to be preserved, since normal inhibition-of-return effects have been observed $[31,39,68]$. In the episodic memory domain, no effect of $\mathrm{AD}$ was found on a retrieval-induced forgetting task [78].

Taken as a whole, these results indicate that not all suppression processes are affected by normal aging or AD. However, at this time, very few studies have explored a series of inhibitory / interference resolution processes in the same group of subjects in order to formally determine the generality of impairments in these populations. In that context, Kramer et al. [66] observed that elderly subjects had more difficulties than young subjects in stopping an overt response and adopting new rules in a categorization task, although both groups produced equivalent negative priming effects, response compatibility effects, spatial precuing effects, and self-reported cognitive failures. More recently, Charlot [20; see also 21] assessed inhibitory / interference resolution functioning in working memory using tasks exploring the access, deletion and restraint functions, and demonstrated that the effects of aging are weaker on the access function than on the other two suppression functions. With regard to AD, Amieva et al. [3] investigated the effects of mild AD on four suppression tasks: the negative priming paradigm, the Stroop test, the go/no-go task and the stop-signal task. Impaired performance was observed on the negative priming and Stroop tasks, but not on the go/no-go task, and only limited impairment was observed on the stop-signal task, suggesting that motor response inhibition could be relatively spared in that group of patients. However, Collette et al. [25] showed a worse performance in AD patients on the go/no-go task, as well as on the Stroop and Hayling tasks. Finally, Belleville et al. [13] assessed verbal suppression processes using the Stroop and the Hayling 
tasks in normal elderly and Alzheimer's disease, and demonstrated impaired performance on both tasks in healthy elderly and patients.

In summary, studies of inhibition / interference resolution abilities in normal aging and AD indicate that, although both populations exhibit deficits on a large range of tasks, they do not present a general suppression dysfunction. In a comprehensive review, Amieva et al. [4] showed that the effect of Alzheimer's disease was obvious on tasks requiring controlled inhibition processes (e.g. the Stroop), but not on tasks requiring more automatic inhibition (e.g. inhibition of return). So, on the basis of the taxonomy proposed by Nigg [81], the authors concluded that AD patients were impaired only on tasks requiring conscious cognitive control. The observation of the performances obtained by elderly subjects evidenced a similar pattern of results, suggesting also a selective deficit of controlled inhibitory mechanisms in normal aging. The only difference observed between the patterns of results associated to each population concerns a specific deficit in normal aging on motor inhibitory tasks. However, tasks used to assess motor inhibition in normal aging studies are characterized by a lower frequency of withholding responses than that administered to AD patients, which would place more substantial demands on controlled inhibition [56]. It seems thus that the pattern of preserved/impaired inhibitory tasks in normal aging can be related, as in AD patients, to a specific impairment of inhibitory processes requiring conscious cognitive control.

To the best of our knowledge, there exists very few studies that explored the specificity of inhibitory / interference resolution dysfunctions in these populations with reference to theoretical models proposed in the literature [see however 20], nor that directly compare the effect of AD and normal aging on suppression abilities [see however 13]. Consequently, the aim of the present study was to formally explore if the pattern of performance presented by elderly subjects and AD patients is in agreement with the theoretical framework proposed by Kipp Harnishfeger [45,46,104], distinguishing between the concepts of inhibition and interference. As indicated previously, inhibitory control corresponds to a voluntary/conscious suppression of the information classified as irrelevant for the ongoing task, and interference resolution represents an automatic gating mechanism occurring prior to conscious awareness and preventing the processing of distracting information. The exploration of the adequacy of this theoretical framework to suppression deficits in normal aging and Alzheimer's 
disease was motivated by two arguments. First, developmental data attests that inhibition and interference resolution mechanisms can be dissociated. Indeed, younger children were less efficient than older children and adults at consciously suppressing information while young and old children and adults showed a similar performance when the task required automatic inhibition of competing items [97]. Second, the performance of elderly subjects and AD patients on various suppression tasks suggest the existence of an impairment of controlled inhibitory processes while more automatic processes are preserved.

Consequently, a series of four tasks requiring either inhibitory or interference processes was administered to young subjects, healthy elderly subjects and AD patients. With regard to interference resolution tasks, a correct performance will be obtained only if subjects prevent other information to interfere with the processing of the target information. In the probe recency task [61], subjects were required to judge whether a probe letter was a member of a set of previously presented items. In some trials, the probe letter was not member of the target set but was presented just before, and thus this distracting information interfered with the recognition process in the current trial. In the flanker task [92], three words were presented (one central target and two flankers), with the instruction to semantically categorize the central word by pressing one of two key-responses. In some trials, the target and distracting information belong to separate target semantic categories, and were thus associated to different key-responses, leading to interference at the level of response production (namely, to suppress the motor response associated to the distracting category). In these two tasks, subjects were not informed of the presence of distracting information on some trials (probe recency vs response incompatibility), and post-hoc questioning confirmed that they were not aware of that experimental manipulation. On the contrary, the exploration of inhibitory control (assessed with a directed forgetting task [84] and the Hayling task [17]) required to actively suppress an information that is or became irrelevant for the ongoing task. In the directed forgetting working memory task, one or two series of letters were successively presented and, on some trials, an instruction to forget was explicitly given following the presentation of the second series. The performance recall for the last series was compared between the different conditions. In the Hayling task, subjects have to complete sentences in which the last word is omitted, but has a particularly high probability of one specific 
response, by any word unrelated to the sentence. So, subjects have to explicitly suppress a mandatory response in order to produce a less automatic response.

The exploration of suppression abilities in normal aging and Alzheimer's disease in relation to the distinction between controlled inhibition and automatic interference resolution [104] appears to be particularly worth investigating. Indeed, a dominant theory to explain cognitive changes associated to normal aging is that the cognitive resources available to perform mental operations decrease with age [82]. Since controlled processes are considered to require more attentional resources than automatic ones, we expect a lower inhibitory performance in the group of elderly (by comparison to young subjects) for the tasks requiring a conscious suppression of some information, but a similar performance in the two groups with regard to tasks requiring automatic interference resolution processes. We also expect a further and specific decrease of performance in AD patients (by comparison to healthy elderly subjects) on inhibitory tasks requiring controlled processes. Indeed, it is now well acknowledged that the various cognitive deficits presented by AD patients in the early stages of the disease are characterized by an impairment of controlled processes associated to a preservation of the automatic ones $[1,38,87]$.

\section{Methods}

\section{Subjects}

Seventy adults volunteered to participate in this study. All subjects had normal or corrected vision and normal or corrected hearing. The 30 younger adults (14 men and 16 women) had an average age of 22.4 years (range $=19-26$ ). The 20 normal elderly subjects ( 5 men and 15 women) had an average age of $72.3 \pm 5.1$ years (range $=63-80$ ). These elderly participants were non-institutionalized, alert, and had no history of neurological problems, alcohol abuse or psychiatric disorders. To assess crystallized verbal ability, each young and elderly participant was administered the Mill-Hill Vocabulary Scale (multiple-choice form; a French-language adaptation [32]). No significant difference was found between the scores of the younger (26.10 \pm 3$)$ and older (27.03 \pm 4.95$)$ subjects $[\mathrm{t}(38)=0.25, \mathrm{p}>.5]$. All participants were native speakers of French and did not report any medical, neurological or sensory defects, or use of medication likely to alter cognitive functioning. 
A total of 20 patients attending the Day Care Center for Memory Disorders in Elderly (CHU Liège) also participated in this study. The patients (6 men and 14 women) met the NINCDS-ADRDA criteria for probable Alzheimer’s disease [76] and suffered from Alzheimer’s disease at a mild stage. All patients had suffered from progressively worse memory problems for at least 6 months. The diagnosis of AD was based on general medical, neurological and neuropsychological examination. Structural neuroimaging showed only slight atrophy or mild leukoaraiosis. Patients' age ranged from 58 to 83 years (mean age: $74 \pm 5.8$ years). No patients suffered from any other medical or neurological condition nor did they take medication that would be likely to adversely affect cognitive performance. They were able to hear and see adequately and follow instructions. The normal elderly subjects were matched as accurately as possible for age, sex and sociocultural level to the AD patients. These control subjects did not differ from AD patients according to age $[\mathrm{t}(38)=0.98, \mathrm{p}>.5]$ or education level $[t(38)=0.33, p>.5]$. The elderly participants and AD patients were also administered the Mattis Dementia Rating Scale [73], which is widely used to screen for dementia. All control subjects had a total score superior to 130 on this scale, which constitutes a cut-off score to discriminate normal aging from dementia [77]. Overall performance on the Mattis dementia rating scale was significantly lower for $\mathrm{AD}$ patients than for control subjects $[\mathrm{t}(38)=9.08, \mathrm{p}<.00001$; $\mathrm{AD}$ patients $=119.6 \pm 8.9$; control subjects $=139.1 \pm 3.3]$.

\section{Procedure}

The participants were tested individually in a two-hour session in a quiet room. Most of the tasks were presented on a microcomputer. In order to avoid practice and weariness effects, two orders for the administration of the tasks were defined and were randomly assigned to participants. Computerized tasks were presented on a PC-compatible computer interfaced with a 14-inch SVGA color monitor using E-Prime software version 1.0 [90]. They were seated in front of the computer screen so that their eyes were approximately $70 \mathrm{~cm}$ from the display.

\section{Cognitive tasks}

Interference control

Probe recency task 
The design of this task was similar to that used in neuroimaging studies by Jonides et al. [61] and D'Esposito et al. [29]. Each subject completed 80 trials of an item recognition memory task (four sessions of 20 trials separated by short periods of rest) in which they were required to judge whether a test probe item was a member of a set of previously studied items. In each trial, four consonants were simultaneously presented in the center of a computer screen for 1,500 milliseconds. After a retention delay of 3,000 milliseconds, the probe letter was presented and the subjects had to decide, and indicate by a key-press, whether the probe was one of the four letters previously presented in that trial. The probe letter remained displayed for a maximum of 2,000 milliseconds or until the subject responded. Practice trials were administered before the beginning of each session. The task was composed of four conditions: (1) Recent Negative trials, in which the probe did not match any items in the target set of the present trial but did match an item from the target set of one of the two previous trials (and thus required a "no" response); (2) Nonrecent Negative trials, in which the probe matched items from neither the current nor the two previous target sets (and thus required a "no" response); (3) Recent Positive trials, in which the probe matched an item that was presented in the current target set (and thus required a "yes” response) and in one of the two previous target sets; and (4) Nonrecent Positive trials, in which the probe matched an item that was presented in the current target set (and thus required a "yes" response) but not in any of the two previous target sets. The four kinds of trials were randomly administered across the four sessions, with the restriction that no more than three trials of the same type were presented in succession (the order of presentation being similar for all subjects). Subjects were not informed about the manipulation of probe recency.

\section{The flanker task}

This task was a French adaptation of that used by Shaw [92]. The material consisted of 16 target words (8 metal words and 8 furniture words) and 16 neutral flanker items (8 vehicles and 8 fishes) taken from Brulex [28] and representing the most frequent category exemplars. The mean number of graphemes in the words in each of the four categories was similar $[F(1,12)=0.05 ; p>.9]$. In each trial, the stimuli were three words (one central target and two flankers) presented on a single column in uppercase letters on a computer screen. The task was described to participants as a study of reading 
and categorization of the centrally presented information and they were told not to pay any attention to the flanker words. The subjects were given 128 trials in four sessions of 32 trials each separated by short rest periods. Practice trials were administered before the beginning of each session. At the outset of each trial, a blinking arrow appeared for 600 milliseconds in the location in which the first letter of the target word was to appear. As the fixation point disappeared, it was immediately replaced by the target and flanker words. These stimuli were removed immediately after the participant responded. On each appearance, the target word was flanked, above and below, by an additional word. Participants pressed one of two response-keys to indicate the category of each target word. Each of the four experimental conditions was determined by the relation between the target and flanker words. For two of the conditions, the target and flanker words were associated with compatible responses (namely, the same response-key): in the same-word (SW) condition, the target word appeared three times on the screen, because the flanker word was identical to the target; in the same-category (SC) condition, the target and flanker words were two exemplars of the same category. In the third, or neutral-response (NR) condition, the flanker was one of the neutral words (and thus was not associated with any response-key). In the different-response (DR) condition, the target and flanker words were from separate target categories and were therefore associated with incompatible responses.

The flanker task requires in fact several suppression processes. Indeed, subjects were given the instruction to ignore the flanker words and to concentrate on the central target word. Although the instruction to ignore some information can be related to controlled inhibitory processes, some data (see Shaw [92]) are indicative that the flanking information is nevertheless processed, leading to a phenomenon of perceptual interference. Moreover, some output interference comes also from the association of the target and flanker words to different key responses in the different-response condition. Controlled inhibition is required in a similar way in each condition, and consequently this effect will disappear with the comparison of the different conditions. On the contrary, the automatic perceptual and output interference effects are more important in the different-response condition, and will be evidenced by the comparison of that condition to the other ones. Subjects were not warned that, in the different-response condition, the relationship between targets and flankers leads to interference at the level of response production. 


\section{Inhibitory control}

\section{Directed forgetting in working memory}

We used a procedure adapted from Reed's [84] study and similar to that used by Andrès et al. [9]. In this task, trigrams composed of consonants were presented for 2,000 milliseconds on a computer screen and subjects were asked to read aloud and memorize the trigrams presented. Three experimental conditions were administered. In the single-trigram condition (control condition), a single trigram (three consonants) was presented for retention. In the retroactive interference condition, a second (interfering) trigram was presented for retention immediately after the first one. In the directed forgetting (or inhibition) condition, two trigrams were also presented consecutively. However, immediately after the presentation of the second trigram, a screen displayed the message "to be forgotten" for 500 milliseconds, which prompted participants to forget the trigram as they would not be required to recall it later. Immediately after the presentation of the trigram(s), an interpolated activity was presented that consisted of reading strings of numbers aloud for 10 seconds. Next, the participants were asked to recall the three letters of the trigram(s), with no time constraint. In the inhibition condition, only the first trigram had to be recalled while in the interference condition, subjects have to recall the first and second trigrams. Three practice trials, one per condition, were given prior to the beginning of the task. Participants were then presented with thirty trials, ten per experimental condition. The trials belonging to the three conditions were presented in an intermixed way, but the same pre-established order was administered to all participants.

A criticism that could be made to the directed forgetting task concerns its susceptibility to proactive interference building across trials. However, since the trials belonging to the different conditions were randomly presented, the susceptibility to proactive interference, even if not similar between the three groups, should be proportionally of the same amplitude for the three conditions. So, proactive interference should not influence the comparison of performance between the three conditions across the groups.

\section{The Hayling task}


This task assesses the capacity to suppress (inhibit) a habitual response and was initially devised to examine both initiation and inhibition processes [17]. The Hayling task consists of 30 sentences in which the final word is omitted but has a particularly high probability of one specific response. The task is composed of two sections (A and B), each containing 15 sentences. In section A (initiation), sentences are read aloud to subjects who have to complete each sentence with the missing word. In section B (response suppression), sentences are read aloud to subjects who this time have to complete the sentence not with the expected word but with a word unrelated to the sentence. If at any time during this stage of the test, subjects give a sentence completion rather than an unrelated word, they are told that the word is too closely related to the sentence, and the task instructions are repeated. If a subject does not produce a word within 30 seconds, that trial is terminated and a response latency of 30 seconds is recorded. Different measures of response suppression abilities were used in the analysis. First, section B latencies minus section A latencies were considered for each subject, which presumably represents the additional thinking time required in having to produce a novel word rather than a straightforward sentence completion. Secondly, a semantic score was devised for section B whereby the overall semantic relationship of each response to its stimulus sentence was measured: three points were given if the word was a straightforward completion of the sentence, one point for a word semantically related to the sentence in some way and no score when the response successfully fulfilled the task requirements (namely, no relationship was found between the word produced and the remaining of the sentence).

\section{Additional measures}

\section{Processing speed}

This task was administered in order to assess general processing speed and examine the possible contribution of a reduction in processing speed to the inhibitory abilities of normal elderly subjects and Alzheimer's patients. Processing speed was assessed with a letter comparison task, which is a computerized version of the task initially proposed by Salthouse and Babcock [86]. The interest of this task is that it induces no distraction from task-irrelevant information, susceptible to deteriorate the speed performance of elderly subjects [71]. Participants were presented with pairs of letters and their 
task was to decide as quickly and accurately as possible whether the letters were the same or different, by pressing a response-key. The test comprised 60 trials, with 30 "same items" and 30 "different items" trials. The selected measure was the mean correct latency for "same items” trials.

\section{$\underline{\text { Digit span task }}$}

Since three of the tasks required maintenance of information in working memory (the probe recency task, the directed forgetting task and the Hayling task), a digit span task was administered to examine the possible contribution of a reduction in short-term memory capacity to the inhibitory abilities of our subjects. Forward digit span was tested in auditory modality. Lists of two to nine digits were read by the examiner at the rate of one digit per second. Three sequences of each length were presented until the subject failed on three sequences with a particular length. The longest sequence correctly recalled on at least two of the three trials represented the subject's digit span.

\section{Pyramid and palm tree test}

This matching task [58] was administered in order to assess semantic memory in Alzheimer's disease and examine the possible contribution of impaired semantic representations to the inhibitory abilities of these patients. Three pictures are simultaneously presented (the target one on the top and two other pictures on the bottom). The task requires the patient to select the one of the two bottom pictures that is connected with the target picture. 52 trials were administered. Measure of performance was the number of correct matching.

\section{Results}

Statistical analyses consisted of three-way ANOVAs on the groups of young subjects, elderly controls and AD patients. Planned comparisons were used to compare (1) the performance of young and elderly subjects and (2) the performance of elderly controls and AD patients. A statistical level of $\mathrm{p}<.05$ was used for all analyses, and logarithmic values were used to reduce between group variability. Inhibitory and facilitation abilities were measured by both response times and response 
accuracy, and the influence of processing speed and span size on these abilities was assessed by covariance analyses.

\section{Additional measures}

With regard to processing speed, mean response times (in ms) for correct responses on "same items" trials were compared between groups. A significant difference between the three groups was found $[\mathrm{F}(2,67)=25.78, \mathrm{p}<.0001]$, with a slowing down in elderly subjects by comparison to young subjects, as well as in AD patients by comparison to matched control subjects (young: $721 \pm 204$; elderly: $859 \pm 181$; AD: $1296 \pm 536$ ). With regard to the digit span, there was also a significant difference between the three groups $[\mathrm{F}(2,67)=12.34, \mathrm{p}<.0001]$, with a higher span in young subjects than in elderly ones, and in elderly controls compared to AD patients (young: $6.2 \pm 0.9$, elderly: $5.5 \pm$ $1.0 ; \mathrm{AD}: 4.8 \pm 1.0)$.

\section{Interference resolution}

\section{Probe recency task}

Mean response times (RT) for correct responses and accuracy of responses in the four conditions (Nonrecent Negative, Recent Negative, Nonrecent Positive, Recent Positive) are presented in Table 1. The critical features of behavioral performance concern the comparison of the Recent Negative trials to the Nonrecent Negative trials (interference effect) and the comparison of the Recent Positive trials to the Nonrecent Positive trials (facilitation effect). Difference scores were computed to test these two effect: [Nonrecent Negative - Recent Negative] for the interference effect and [Nonrecent Positive Recent Positive] for the facilitation effect.

\section{[Insert Table 1 near here]}

The comparison of the interference effect on RT between the three groups demonstrated no significant differences $[\mathrm{F}(2,67)=0.57, \mathrm{p}>0.5]$, and this was confirmed by planned comparisons [young vs elderly: $\mathrm{F}(1,67)=0.34, \mathrm{p}>0.5$; elderly vs $\mathrm{AD}$ : $\mathrm{F}(1,67)=0.18, \mathrm{p}>0.5]$. Similar results were observed 
for response accuracy [comparison between the three groups: $F(2,67)=1.34, p>0.1$; young vs elderly: $\mathrm{F}(1,67)=1.10, \mathrm{p}>0.1$; elderly vs $\mathrm{AD}: \mathrm{F}(1,67)=0.22, \mathrm{p}>0.5]$.

With regard to the facilitation effect on response times, although no significant difference was found between the three groups $[\mathrm{F}(2,67)=2.83, \mathrm{p}>0.05]$, planned comparisons demonstrated a larger facilitation effect for AD than for elderly controls only [elderly vs $A D$ : $F(1,67)=4.62, p<0.05$; young vs elderly: $F(1,67)=0.10, p>0.7]$. This effect could however be explained by span size and processing speed, since the addition of the covariates into the analysis suppressed the differential facilitation effect between elderly adults and $\mathrm{AD}$ patients $[\mathrm{F}(1,66)=3.56, \mathrm{p}>0.05 ; \mathrm{F}(1,66)=1.66, \mathrm{p}>0.1$, respectively]. Finally, similar analyses were done to determine the effect of facilitation on response accuracy. No significant differences between the three groups was found for accuracy of responses $[\mathrm{F}(2,67)=2.11, \mathrm{p}>0.1]$ and this was confirmed by planned comparisons [young vs elderly subjects $[F(1,67)=0.59, \mathrm{p}>0.1$; elderly vs $\mathrm{AD} F(1,67)=1.37, \mathrm{p}>0.1]$

\section{Discussion}

With regard to the probe recency task, interference effects were observed both for responses times and accuracy of responses. These interference effects were similar in size in all three groups of subjects. This task was previously used by Jonides et al. [61] who found interference effects that were greater in the elderly than the young subjects, indicating an interference resolution deficit. However, unlike to our study, recent Negative and Nonrecent Negative items were not randomly presented but were presented in blocks composed of half recent and half Nonrecent Negatives items, or Nonrecent Negative items only. Moreover, a combined score with response latency and accuracy was used to compare the performance of young and elderly subjects, while we preferred to use two scores to determine whether specific patterns of deficits could be observed with regard to RTs or response accuracy. Such methodological differences could explain the different pattern of results observed between these two studies. With regard to AD, no specific inhibitory deficit was observed. One could argue that this might be due to very poor performance by these patients in all task conditions, or to a rapid decrease in the traces left by previous items, leading to less sensitivity to interference because these items had already completely disappeared from working memory. However, the AD patients' performance was superior to $75 \%$ in all conditions; this is considerably above the chance level. 
Moreover, the presence of a greater facilitation effect suggests the persistence of a memory trace from the previous trials that influences the production of the response. So we can be confident that AD patients present similar interference resolution abilities to elderly subjects in this task.

\section{The flanker task}

Statistical analyses were performed by grouping together the two facilitation conditions (same word and same category) involved in Shaw's [92] initial task. This was done to obtain measures of semantic interference and facilitation that could easily be compared to those obtained in the probe recency task. The critical features of behavioral performance concern the comparison of the different response condition to the neutral response condition (interference effect) and the same word and same category conditions to the neutral response condition (facilitation effect). Difference scores were computed to test these effect: [different response - neutral] for the interference effect and [same word/category neutral] for the facilitation effect. Table 2 shows average response time and number of correct responses as a function of group and condition.

\section{[Insert Table 2 near here]}

The comparison of the interference effect on response times demonstrated no significant differences between the three groups $[\mathrm{F}(2,67)=1.93, \mathrm{p}>0.1]$, that was confirmed by planned comparisons [young vs elderly $\mathrm{F}(1,67)=1.14, \mathrm{p}>0.1$; elderly $v s \mathrm{AD} F(1,67)=0.62, \mathrm{p}>0.1$ ]. With regard to the facilitation effect, again no significant differences between the three groups was observed $[F(2,67)=2.66, \mathrm{p}>0.05]$. However, planned comparisons demonstrated a larger facilitation effect in AD subjects [young vs elderly: $\mathrm{F}(1,67)=1.36, \mathrm{p}>0.1$; elderly vs $\mathrm{AD}$ : $\mathrm{F}(1,67)=5.31, \mathrm{p}<0.05$ ]. However, when span size and speed of processing were taken as confounding covariates, this effect disappeared $[F(1,66)=4.02, p>0.05 ; F(1,66)=1.98, p>0.1$, respectively $]$.

With regard to measure accuracy, the comparison of the three groups demonstrated a similar interference effect $[F(2,67)=2.92, p>0.05]$. However, planned comparisons demonstrated that, while there was no difference between young and elderly subjects $[F(1,67)=0.63, p>0.1]$, AD patients 
presented a larger interference effect than elderly subjects $[F(1,67)=5.49, p<0.05]$. This effect remains significant when span size and processing speed were used as covariates $[\mathrm{F}(1,66)=4.75, \mathrm{p}<0.05$; $F(1,66)=4.26, p<0.05$, respectively]. Finally, the comparison of the facilitation effect on accuracy of responses demonstrated a significant difference between the three groups $[F(2,67)=5.47, p<0.01]$, with a similar effect between young and elderly subjects $[F(1,67)=1.14, p>0.1]$, but a larger facilitation effect in $\mathrm{AD}$ patients than elderly subjects $[\mathrm{F}(1,67)=4.86, \mathrm{p}<0.05]$. This effect disappeared when span size and processing speed were used as covariates $[\mathrm{F}(1,66)=3.22, \mathrm{p}>0.05 ; \mathrm{F}(1,66)=3.89, \mathrm{p}>0.05$, respectively].

\section{Discussion}

The flanker task [92] requires to process only the centrally presented information and, in some trials, the relationship between targets and flankers leads to interference at the level of perceptual input and response production. The flanker task did not demonstrate any sensitivity to interference in normal aging or in $\mathrm{AD}$, when assessed by RTs, but a greater sensitivity to interference in $\mathrm{AD}$ patients when assessed by response accuracy. Shaw used this task to show that elderly subjects present greater semantic inhibitory effects than young subjects but equivalent semantic facilitation in the time taken to classify target words. However, semantic inhibition deficits were not systematically observed in normal aging [see, for example, 55,83]. With regard to AD, a slight impairment was found for response accuracy, but not for RTs, and this impairment was not explained by a reduction of span size or a slowing down. In order to test if the inhibitory effect cannot be explained by the semantic deficits frequently observed in the disease (for a review, see [43]), correlations were computed between performance in the different conditions of the flanker task and performance on the designation task “Pyramid and Palm Tree test” [58]. No significant correlation was found (all p>0.05), indicating an absence of relationship between the performance on the flanker task and the semantic abilities of the $\mathrm{AD}$ patients. So, results obtained are in agreement with the hypothesis that there exists some difficulties in Alzheimer's disease (expressed by a measure of accuracy only) to control interference coming from distracting information in a semantic decision task. 


\section{Inhibitory control}

\section{Directed forgetting}

Participants' responses were scored by assigning one point for each letter recalled (regardless of its position within the trigram) (maximum score per condition was therefore 30). In the retroactive interference condition (i.e., presentation of a second, interfering trigram), only recall of the first trigram was scored (as in the inhibition condition). Sensitivity to retroactive interference was measured by the difference in performance between the single-trigram and retroactive interference conditions. Inhibitory capacity was measured by the difference in recall performance between the retroactive interference and directed forgetting conditions (see Table 3). This measure was preferred to the one used by Andrès [9] (namely, a comparison between the directed forgetting and control conditions) in order to suppress a putative effect of retroactive interference on the measure of inhibition.

\section{[Insert Table 3 near here]}

A 3 (group: young, elderly, AD) X 3 (condition: control, retroactive interference, inhibition) ANOVA was done, and demonstrated a significant group effect $[F(2,67)=18.49, \mathrm{p}<.0001]$, with a similar performance for young and elderly, and a lower performance for AD; a significant condition effect $[\mathrm{F}(2,134)=133.39, \mathrm{p}<.0001]$, with a better performance in the single trigram condition than in the retroactive interference and inhibition conditions; and a significant interaction between group and condition $[\mathrm{F}(4,134)=11.35, \mathrm{p}<.0001]$. Planned comparisons revealed a similar decrease in performance between the single trigram and the retroactive interference condition in young and elderly subjects, and a larger decrease in $\mathrm{AD}$ patients than elderly. With regard to the comparison of the retroactive interference and inhibition conditions, planned comparisons demonstrated a greater increase in performance in young subjects than in elderly subjects, but a similar pattern of performance in the elderly and $\mathrm{AD}$ groups (namely, no improvement in performance from the retroactive interference to the inhibition condition). When span size and processing speed were taken as confounding covariates, the interaction effect remained significant $[$ span size: $F(4,132)=9.08 p<$ 
.0001 ; processing speed: $\mathrm{F}(4,132)=6.51, \mathrm{p}<.0001]$. A directed forgetting score was also calculated by subtracting the performance on the directed forgetting condition from that on the retroactive interference condition. As observed with the ANOVA, between groups differences were also found $[\mathrm{F}(2,67)=3.51, \mathrm{p}<0.05]$, with elderly subjects having a larger directed forgetting effect than young subjects $[F(1,67)=6.55, \mathrm{p}<0.05]$, while a similar effect was found between elderly subjects and AD patients $[\mathrm{F}(1,67)=0.68, \mathrm{p}>0.1]$. When span size and processing speed were taken as confounding covariates, the interaction effect remained significant [span size: $\mathrm{F}(1,66)=11.94 \mathrm{p}<.0001$; processing speed: $\mathrm{F}(1,66)=15.02, \mathrm{p}<.0005]$.

Finally, the different types of errors were analyzed. Significant differences were found for the omission errors (the number of consonants missing) $[\mathrm{F}(2,67)=13.94, \mathrm{p}<.0001]$, with no differences between young and elderly subjects but a difference between elderly subjects and AD patients. This difference remained significant when span size and processing speed were used as confounding covariates [respectively, $\mathrm{F}(2,66)=7.13, \mathrm{p}<.005 ; \mathrm{F}(2,66)=5.99, \mathrm{p}<.005]$. Similarly, the number of to-be-forgotten (TBF) errors (corresponding to the number of consonants produced that belonged to the second trigram in the inhibition condition) differs between the three groups $[F(2,67)=29.74, p<$ .0001]. Again, young and elderly subjects performed similarly, but AD patients produced more errors than elderly controls. Using span size and processing speed as confounding covariates did not remove the difference between elderly subjects and $\mathrm{AD}$ patients [respectively, $\mathrm{F}(2,66)=7.54, \mathrm{p}<.005$; $F(2,66)=15.18, p<.0001]$. Finally, there was no significant difference between the three groups with regard to the position errors (the number of consonants recalled in an incorrect serial position) $[F(2,67)$ $=1.32, \mathrm{p}>.1]$.

\section{Discussion}

Directed forgetting (as reflected by the difference in correct recall performance between the inhibition and retroactive interference conditions) was more efficient in young than in elderly subjects (and was not explained by a difference in span size or speed of processing), but no difference was found between elderly controls and AD patients. However, AD patients made more omission and intrusion errors than elderly subjects. The existence of an impaired directed forgetting effect in normal aging had already been reported by Andrès et al. [9]. The results obtained in our study indicate that 
elderly participants and $\mathrm{AD}$ patients were less able than young subjects to inhibit no longer relevant information. Moreover, AD patients presented specific difficulties in comparison to normal elderly subjects in recalling information, in the sense that they produced more intrusions of the TBF trigrams, and that these errors were not explained by span size or speed of processing. It has frequently been reported that $\mathrm{AD}$ patients produce more intrusions [2,12,19,69] and perseveration errors [41,42,91] during word recall tasks. Thus, we can suggest that, unlike normal elderly subjects who only have difficulties completely suppressing TBF items from working memory but clearly distinguish between TBF and TBR items (since they produced no more intrusions than young subjects), AD patients have additional difficulties inhibiting the production of the TBF items. We can hypothesize that the memory traces for TBF items are as strongly activated as those of the TBR items and that, due to the source memory deficit presented by these patients (e.g., [79]), the presentation of the recall cue triggers the production of any activated item. Indeed, an influence of source memory failures on the performance in verbal working memory tasks was previously described [see 54] .

\section{The Hayling task}

Measures of initiation of response consisted of mean response times and number of errors in part A of the task. Measures of inhibition consisted in the raw response latency (mean latencies across 15 trials) when the suppression time was controlled for the initiation time (namely, RTs for part B - part A) and the semantic score (measuring the overall semantic relatedness of the responses to the sentence). The results for the three groups of subjects are presented in Table 4.

\section{[Insert Table 4 near here]}

The comparison of response time for Part A (requiring only initiation of response) showed a significant difference between the three groups $[\mathrm{F}(2,67)=7.60, \mathrm{p}<.005]$, with slower RTs for elderly than young subjects and no differences between elderly subjects and AD patients. However, the effect disappeared when span size and processing speed were taken as confounding covariates [respectively, $\mathrm{F}(2,66)=2.86, \mathrm{p}>.05 ; \mathrm{F}(2,66)=1.95, \mathrm{p}>.1]$. With regard to the inhibition portion, the comparison 
of RTs (B-A) revealed significant differences between groups $[\mathrm{F}(2,67)=18.73, \mathrm{p}<.0001]$, with elderly subjects having slower RTs than young subjects, but AD patients performing similarly to elderly subjects. These results were not modified when span size and processing speed were used as covariates [respectively, $\mathrm{F}(2,66)=13.52, \mathrm{p}<.0001 ; \mathrm{F}(2,66)=11.65, \mathrm{p}<.0001$ ].

With regard to error score, very few errors were committed in the Part A. However, the semantic relatedness of responses made in Part B differed for the three groups $[F(2,67)=24.81, p<.0001]$, with elderly subjects erroneously providing more related responses to the stimuli than young subjects and $\mathrm{AD}$ patients providing more related responses than elderly subjects. When span size was taken as a

confounding covariate, the difference between young and elderly subjects remained significant $[F(1,66)=10.32, \mathrm{p}<0.0001]$ but the difference between $\mathrm{AD}$ patients and elderly subjects became marginally significant $[\mathrm{F}(1,66)=3.65, \mathrm{p}=0.06]$. Similarly after controlling for processing speed, the difference in semantic relatedness between elderly subjects and $\mathrm{AD}$ patients remained significant $[F(1,66)=11.29, p<.005]$, but disappeared between young and elderly subjects $[F(2,66)=2.98, p>$ $.05]$.

\section{Discussion}

Deficits on the Hayling task, not totally explained by span size or speed of processing, were observed in elderly subjects for both RT and semantic relatedness of response. AD patients made more errors than elderly in terms of the semantic relatedness of responses, but this difference became marginally significant after the span size was controlled for. Again, deficits of the patients on this task cannot be related to a potential influence of semantic memory impairment on performance, since no significant correlations were observed between measures on the Hayling task and performance on the Pyramid and Palm Tree test (all $\mathrm{p}>$.05). Impaired performance on the Hayling task in normal aging had previously been described in the literature $[6,13,14]$, and was also partly explained by an influence of processing speed [6]. With regard to the AD patients' performance, there were no differences in comparison to control subjects in terms of response speed, but they produced more responses related to the item to be inhibited (for similar results, see [23]). These results indicate that $\mathrm{AD}$ patients are able to correctly inhibit the target word but not to restrain the production of the first alternative responses that came in mind, these alternatives being or not semantically related to the target words. 


\section{General discussion}

The aim of our study was to explore the performance of young volunteers, normal elderly subjects and $\mathrm{AD}$ patients on a series of suppression tasks assessing interference resolution or active inhibitory control. Very few earlier studies had been interested in evaluating the existence of specific inhibitory / interference resolution dysfunction simultaneously in normal and pathological aging, and those studies did not select suppression tasks with reference to recently proposed theoretical frameworks. In that context, we will first briefly discuss the influence of processing speed and working memory capacity on inhibitory abilities. Next, the main question of this study will be addressed, namely if the pattern of performance observed in normal elderly and AD patients can be interpreted with reference to Wilson and Kipp Harnishfeger's [104] hypothesis that controlled inhibition and interference resolution are dissociable cognitive processes.

The results obtained with the battery of tasks are summarized in Table 5 and demonstrate the existence of a dysfunction of suppression abilities in both normal elderly and AD patients. Interestingly, not all tasks were impaired in these two groups. Some authors have proposed that suppression dysfunction in aging represents a side-effect of a general slowing down of information processing (see [101] for the Stroop task). Thus, a measure of processing speed was included in our battery to test that hypothesis. Moreover, participants' digit span size was also assessed since it had been proposed that working memory resources are a mediator between age and decreased performance on complex cognition tasks (e.g. [82,100]) and three of the suppression tasks required to maintain information in short-term memory. Interestingly, the suppression deficits presented by normal elderly and AD subjects were not influenced by these two variables, except for the semantic relatedness score on the Hayling task. The absence of any influence by these variables on suppression performance is not due to a lack of sensitivity of the measures, since processing speed and span size are clearly related to measures that assess facilitation effects. Consequently, the impaired performance of elderly and AD subjects can be explained in terms of inhibitory / interference resolution dysfunction, and not as a consequence of slowing down or a reduction of resources in working memory. On the contrary, the facilitation effects we observed disappear when covariates assessing more general aspects of cognition 
(speed of processing and short-term memory capacity) were used, indicating that these effects cannot be clearly related to the suppression aspects of the tasks only.

[Insert Table 5 near here]

The pattern of performance observed on the battery of tasks is in accordance with the hypothesis that the dysfunction of suppression abilities in normal aging and $\mathrm{AD}$ is not generalized. More specifically, the aim of this study was to explore whether suppression deficits associated with normal aging and $\mathrm{AD}$ can be interpreted in the theoretical framework distinguishing between the concepts of interference resolution and controlled inhibition. Normal elderly subjects clearly demonstrated performances similar to that of young subjects on the two tasks assessing interference resolution but impaired performances when active inhibitory control is required. However, results obtained by $\mathrm{AD}$ patient can not be explained in that theoretical framework. Indeed, interference resolution was preserved in the working memory task while deficits were observed in the flanker task for response accuracy. With regard to the active inhibitory control, the directed forgetting task demonstrated the presence of difficulties to inhibit the production of items that were clearly labeled as "to forget", and this although AD patients present a normal performance on the measure reflecting inhibitory control (the directed forgetting effect). Performance was also altered on the Hayling task, mainly for the error score, that was interpreted as reflecting difficulties to restrain the production of responses strongly activated but that are not totally relevant for the ongoing task.

Our results with normal aging can be interpreted as reflecting a specific impairment of active inhibitory control processes, associated to a preservation of the mechanism of interference resolution. If we consider that interference resolution in the working memory task is a relatively automatic inhibitory process, these results are similar to those reported in earlier studies that distinguished between controlled and automatic inhibitory processes in normal aging. Andrès [7] administered to young and elderly subjects the Stroop interference task (in which subjects have to consciously suppress the prepotent process of reading in order to produce a response) and a negative priming task (supposed to involve automatic inhibitory processes, since subjects were not warned of the 
relationship between the prime and probe trials). The results indicated an age-related effect on the Stroop task but not on the negative priming task. A similar pattern was observed when performance on inhibition of return (requiring automatic inhibitory processes) was contrasted to that obtained in an antisaccade task (in which subjects are explicitly warned to ignore a spatial cue to orient their gaze towards the opposite side of the screen) [8]. This selective impairment of controlled inhibitory processes in normal elderly subjects is consistent with some recent theories of cognitive aging. Indeed, one major theory is that the cognitive resources available to perform mental operations decline with aging [82], leading to greater age-related differences on tasks that require controlled processes than on those requiring automatic processes [50,60,70,99].

The pattern of results presented by AD patients is more difficult to interpret in the context of a theoretical distinction between inhibitory control and interference resolution. Indeed, normal performance is observed both for the directed forgetting task and for the probe recency task. One common characteristic of these two tasks is that the information to suppress is not present when the subject produce the response: this information was present in the one of the two previous trials in the interference resolution tasks and was presented 10 sec. before the presentation of the response in the directed forgetting task. On the contrary, information to suppress remained present in the two tasks impaired in AD patients: the distracting information remained on the screen until the production of a response in the flanker task and we consider that, in the Hayling task, the word to inhibit, as well as the remaining of the sentence is accessible to consciousness during the search for a semantically unrelated item. So, we can hypothesize that, although AD patients are not able to prevent the processing of distracting information (as evidenced in the Hayling and flanker tasks), these patients are not sensitive to interference from information that was previously processed (as it is the case in the directed forgetting and interference resolution tasks).

The pattern of results we obtained in AD fit particularly well with the proposal of Houghton and Tipper [56]. These authors proposed that the strength of the cognitive processes or representations that have to be suppressed will determine the degree of effortfulness of the mechanisms required to suppress it. In other words, these authors suggest that a very skilled process (e.g. reading for a highschool level adult) or a representation strongly activated (for example, following repeated exposures) 
will require more cognitive resources to be suppressed than few skilled processes or less activated representations. Due to their memory problem, AD patients process information less deeply than normal elderly [103], or they forget more quickly the information previously encoded (see for a review [85]). Consequently, the memory traces on which suppression will apply will be less strong in AD patients and suppression of that information will require less effort than for normal elderly subjects. So, a similar performance will be observed, not because AD patients have preserved suppression abilities but because their residual suppression abilities are sufficient to process information whose traces were partially decayed. On the contrary, when the information that must be suppressed is present, suppression abilities of $\mathrm{AD}$ patients do not allow to prevent the processing of that information. Consequently, the patients present a larger interference / inhibitory effect than healthy elderly. An indirect confirmation of this interpretation is provided by the presence of a larger facilitation effect in $\mathrm{AD}$ when the distracting information in the flanker task belong to the same category than the information to suppress. Nevertheless, the design of the present study does not allow to specifically test the relationship between the strength of the memory traces and the degree of suppression necessary to suppress it. So, this interpretation remains tentative and further studies will be necessary to confirm it.

This distinction between an impairment on the suppression of distracting information in the environment but preserved suppression abilities for information previously processed in AD patients can also be tentatively related to the concepts of "access" and "deletion" functions as proposed by Hasher et al. [52]. In the present study, interference resolution in the probe recency and the flanker tasks can be considered as depending on the access function; inhibition in the directed forgetting task as depending on the deletion function; and inhibition in the Hayling task as depending on the restraint function. Our results indicate that, by comparison to elderly subjects, AD patients are no more impaired than elderly subjects on the deletion function, but present a decreased performance when the restraint function was assessed. Nevertheless, the performance on the two interference resolution tasks does not allow to determine if there exists a deficit of the deletion function, since a normal performance on the probe recency task was associated to a decreased performance on the flanker task. So, our data concerning suppression abilities in AD are not completely in agreement with the 
theoretical framework proposed by Hasher et al. [52]. It must nevertheless be emphasized that the pattern of results observed in elderly (that was previously interpreted as reflecting a specific impairment of controlled inhibitory processes) is also clearly in agreement with the proposal of Hasher et al., and is indicative of a specific preservation of the access function in normal aging.

In conclusion, this study revealed that distinct suppression deficits are observed in normal aging and Alzheimer's disease. By comparison to young subjects, normal elderly subjects demonstrate a specific impairment of suppression processes requing an active inhibition of some information, but the ability to resolve interference from distracting information appears preserved. Otherwise, the comparison of performance in elderly subjects and $\mathrm{AD}$ patients seems to indicate that suppression processes are quantitatively less efficient in $\mathrm{AD}$, since these patients present a correct suppression performance only when the trace of information is partially decayed. This could indicate that the impaired performance on suppression tasks in $\mathrm{AD}$ does not represent a specific alteration of some inhibitory / interference resolution processes (as observed in normal aging) but rather a less efficient functioning of all these processes. From a theoretical point of view, results of this study are indicative that more attention should be paid to the integration of the different inhibitory frameworks proposed in the litterature. Indeed, the design of this study was build up to determine if suppression deficits reported in normal aging and Alzheimer can be interpreted as reflecting a deterioration of controlled inhibitory processes associated to a preservation of more automatic interference resolution processes [104]. Although in agreement with our initial hypothesis, the pattern of results observed in normal aging, also fits with the proposal of three distinct suppression functions in working memory [52]. 


\section{ACKNOWLEDGMENTS}

This work was supported by the Belgian National Fund for Scientific Research (FNRS), the Interuniversity Attraction Poles Program P5/04, Belgian Science Policy and the Government of the French-Speaking Community of Belgium (Actions de Recherche Concertées, Grant 05-10-332) F. Collette and C. Schmidt are respectively Research Associate and Doctoral Researcher at the FNRS. Disclosure statement: all authors disclose any actual or potential conflicts of interest including any financial, personal or other relationships with other people or organizations within three years of beginning the work submitted that could inappropriately influence the work. 


\section{REFERENCES}

1. Adam S, Van der Linden M, Collette F, Lemauvais L, Salmon E, Further exploration of controlled and automatic processes in early Alzheimer's disease. Neuropsychol 2005;19:420-427.

2. Amieva H, Lafont S, Rainville C, Dartigues J-F, Fabrigoule C. Analyse of inhibitory dysfunction in patients with Alzheimer's disease and normal elderly adults in two verbal tasks. Brain Cogn 1998;37:58-60.

3. Amieva H, Lafont S, Auriacombe S, LeCarret N, Dartigues J-F, Orgogozo J, Fabrigoule C. Inhibitory breakdown and dementia of the Alzheimer type: A general phenomenon? J Clin Exp Neuropsychol 2002;24:503-516.

4. Amieva H, Phillips LH, Della Sala S, Henry JD. Inhibitory functioning in Alzheimer's disease: A review. Brain 2004;127:949-964.

5. Amieva H, Lafont S, Rouch-Leroyer I, Rainville C, Dartigues J-F, Orgogozo J, FabrigouleC. Evidencing inhibitory deficits in Alzheimer's disease through interference effects and shifting disabilities in the Stroop test. Arch Clin Neuropsychol 2004;19:791-803.

6. Andrès P, Van der Linden M. Age-related differences in supervisory attentional system functions. J Gerontol: Psychol Sci 2000;55B,P373-P380.

7. Andrès P. Ageing and inhibition: distinguishing between automatic and controlled inhibition. Paper presented at the Annual Conference of the British Psychological Society, Blackpool, 2002.

8. Andrès P. Further evidence for the fractionation of inhibition in ageing. Paper presented at the Annual Conference of the British Psychological Society, Bournemouth, 2003.

9. Andrès P, Van der Linden M, Parmentier FB. Directed forgetting in working memory: age-related differences. Memory 2004;12:248-256.

10. Arbuthnott K. Inhibitory mechanisms in cognition: Phenomena and models. Cur Psychol Cogn 1995;14:3-45.

11. Balota DA, Faust M. Attention in dementia of the Alzheimer's type. In : Boller F., Grafman J., editors. Handbook of Neuropsychology, $2^{\text {nd }}$ Edition, Vol. 6. Amsterdam: Elsevier Science ; 2001, 
p. $51-80$.

12. Bandera L, Della Sala S, Laiacona M, Luzzatti C, Spinnler H. Generative associative naming in dementia of Alzheimer's type. Neuropsychologia 1991;29:291-304.

13. Belleville S., Rouleau N, Van der Linden, M. Use of the Hayling task to measure inhibition of prepotent responses in normal aging and Alzheimer’s disease. Brain Cogn 2006;62:113-119.

14. Bielak AA, Mansuetti L, Strauss E, Dixon RA. Performance on the Hayling and Brixton tests in older adults: norms and correlates. Arch Clin Neuropsychol 2006;21:141-149.

15. Bjorklund DF, Harnishfeger KK. The evolution of inhibitionmechanisms and their role in human cognition and behavior. In Dempster FN, Brainerd CJ, Editors. Interference and inhibition in cognition. San Diego: Academic Press ; 1995, p. 142-173.

16. Bondi MW, Serody AB, Chan AS, Eberson-Shumate SC, Delis DC, Hansen LA, Salmon DP. Cognitive and neuropathologic correlates of Stroop color-word test performance in Alzheimer's disease. Neuropsychol 2002;16:335-343.

17. Burgess PW, Shallice T. Response suppression, initiation and strategy use following frontal lobe lesions. Neuropsychologia 1996;34:263-273.

18. Butler KM, Zacks RT, Henderson JM. Suppression of reflexive saccades in younger and older adults: Age comparisons on an antisaccade task. Memory Cogn 1999;27:584-591.

19. Cahn DA, Salmon DP, Bondi MW, Butters N, Johnson SA, Wiederholt WC, Barret-Connor E.. A population-based analysis of qualitative features of the neuropsychological test performance of individuals with dementia of the Alzheimer type: implications for individuals with questionable dementia. J Int Neuropsychol Soc 1997;3:387-393.

20. Charlot V, Feyereisen P. Aging and the deletion function of inhibition. Aging, Neuropsychol Cogn 2004;11:12-24.

21. Charlot V, Feyereisen P. Mémoire épisodique et déficit d'inhibition au cours du vieillissement cognitif: un examen de l'hypothèse frontale. L'Année Psychologique 2005;105:323-357. 
22. Clark JM. Contributions of inhibitory mechanisms to unified theory in neuroscience and psychology. Brain Cogn 1996;30:127-152.

23. Collette F, Van der Linden M, Salmon E. Executive dysfunction in Alzheimer's disease. Cortex 1999;35:57-72.

24. Collette F, Van der Linden M. Attention disorders in degenerative syndromes. In : Leclercq M, Zimmerman P, editors. Applied neuropsychology of attention: Theory, diagnosis and rehabilitation. London: Psychology Press; 2002, p. 305-338.

25. Collette F, Van der Linden M, Delrue G, Salmon E. Frontal hypometabolism does not explain inhibitory dysfunction in Alzheimer's disease. Alzh Disease Associated Disorders 2002;16:228238.

26. Connelly SL, Hasher L, Zacks R. Age and reading: The impact of distraction. Psychol Aging 1991;6:533-541.

27. Connelly L, Hasher L. Aging and the inhibition of spatial location. J Exp Psychol : Hum Percept Perf 1993;19:1238-1250.

28. Content A, Mousty P, Radeau M. Brulex: une base de données lexicales informatisée pour le français écrit et parlé. L'Année Psychologique 1990;90:551-566.

29. D'Esposito M, Postle BR, Jonides J, Smith EE. The neural substrate and temporal dynamics of interference effects in working memory as revealed by event-related functional MRI. Proc Nat Acad Sci 1999;96:7514-7519.

30. Dagenbach D, Carr TH. Inhibitory processes in attention, memory and language. San Diego: Academic Press; 1994.

31. Danckert, J, Maruff P, Crowe S, Currie J. Inhibitory processes in covert orienting in patients with Alzheimer's disease. Neuropsychol 1998;12:225-241.

32. Deltour JJ. Echelle de vocabulaire Mill Hill de J.C. Raven. Braine-le-Chateau, Belgium: Editions l'Application des Techniques Modernes; 1993 
33. Dempster FN, Brainerd CJ. Interference and inhibition in cognition. San Diego: Academic Press, 1995.

34. Dempster FN, Corkill AJ. Individual differences in susceptibility to interference and general cognitive ability. Acta Psychologica, 1999;101:395-416.

35. Dempster FN, Corkill AJ. Interference and inhibition in cognition and bahavior: Unifying themes for educational psychology. Educat Psychol Rev 1999;11:1-88.

36. Duchek J, Balota DA, Thessing VC. Inhibition of visual and conceptual information during reading in healthly aging and Alzheimer's disease. Aging Neuropsychol Cogn 1998;5:169-181.

37. Dulaney CL, Rogers WA. Mechanisms underlying reduction in Stroop interference with practice for young and old adults. J Exp Psychol: Learn Memory Cogn 1994;20:470-484.

38. Fabrigoule C, Rouch I, Letenneur L, Commenges D, Mazaux JM, Orgogozo JM, Dartigues JF. Cognitive process in preclinical phase of dementia. Brain 1998;121:135-141.

39. Faust ME, Balota DA. Inhibition of return and visuospatial attention in healthy older adults and individuals with dementia of the Alzheimer type. Neuropsychol 1997;11:13-29.

40. Fisher LM, Freed DM, Corkin S. Stroop color-word test performance in patients with Alzheimer's disease. J Clin Exp Neuropsychol 1990;12:745-758.

41. Fox LS, Olin JT, Erblich J, Ippen CG, Schneider LS. Severity of cognitive impairment in Alzheimer's disease affects list learning using the California Verbal Learning Test (CVLT). Int J Geriatric Psych 1998;13:544-549.

42. Fuld P, Katzman R, Davies P, Terry RD. Intrusions as a sign of Alzheimer dementia: chemical and pathological verification. Ann Neurol 1982;11:155-159.

43. Garrard P, Patterson K, Hodges JR. Semantic processing in Alzheimer's disease. In : Morris R, Becker J, editors. Cognitive neuropsychology of Alzheimer's disease. Oxford: Oxford University Press; 2004, p. 179-196.

44. Hamm VP, Hasher L. Age and the availability of inferences. Psychol Aging. 1992;7:56-64. 
45. Harnishfeger KK, Bjorklund DF. The ontogeny of inhibitory mechanisms: A renewed approach to cognitive development. In: Howe ML, Pasnak R, editors. Emerging themes in cognitive development. Vol 1. Foundations. New-York: Springer-Verlag; 1993, p. 28-49.

46. Harnishfeger K. The development of cognitive inhibition. Theories, definitions, and research evidence. In : Dempster FN, Brainerd CJ, editors. Interference and inhibition in cognition. London: Academic Press; 1995, p. 175-206.

47. Hartley AA. Evidence for the selective preservation of spatial selective attention in old age. Psychol Aging 1993;8:371-379.

48. Hartley AA, Kieley JM. Adult age differences in the inhibition of return of visual attention. Psychol Aging 1995;10:670-684.

49. Hartman M, Hasher L. Aging and suppression: Memory for previously relevant information. Psychol Aging 1991;6:587-594.

50. Hasher L, Zacks R. Automatic and effortful processes in memory. J Exp Psychol: Gen 1979;108:356-388.

51. Hasher L, Stoltzfus ER, Zacks RT, Rypma B. Age and inhibition. J Exp Psychol: Learn Mem Cogn 1991;17:163-169.

52. Hasher L, Zacks RT, May CP. Inhibitory control, circardian arousal, and age. In : Gopher D, Koriat A, editors. Attention and Performance XVII, Cognitive Regulation of Performance: Interaction of Theory and Application. Cambridge, MA: MIT Press; 1999, p. 653-675.

53. Hasher L, Tonev ST, Lustig C, Zacks R. Inhibitory control, environmental support, and selfinitiated processing in aging. In : Naveh-Benjamin M, Moscovitch M, Roediger HLI, editors. Perspectives on human memory and cognitive aging. Essays in honour of Fergus Craik New York: Psychology Press; 2001, p. 286-297.

54. Hedden T, Park DC. Contributions of source and inhibitory mechanisms to age-related retroactive interference in verbal working memory. J Exp Psychol: General 2003;132:93-112. 
55. Hogge M, Adam S, Collette F. Retrieval-induced forgetting in normal aging. In: Casini A, Klein O, editors. Proceedings of the 2004 Annual. Meeting of the Belgian Psychological Society Brussels University Press: Bruxelles (Belgium); 2004, p. 63.

56. Houghton G, Tipper SP. A model of inhibitory mechanisms in selective attention. In Dagenbach D, Carr TH, editors. Inhibitory processes in attention, memory, and language. San Diego: Academic Press; 1994, p. 53-112.

57. Houx PJ, Jolles J, Vreeling FW. Aging effects assessed with the Stroop color word test in childhood, adulthood, and aging. Exp Aging Res 1993;19:209-224.

58. Howard D, Patterson K. Pyramids and palm trees: a test of semantic access from pictures and words. Bury St Edmonds: Thames Valley Company; 1992.

59. Intons-Peterson MJ, Rocchi P, West T, McLellan K, Hackney A. Aging; optimal testing times, and negative priming. J Exp Psychol: Learn Mem Cogn 1998;24:362-376.

60. Jennings JM, Merikle PM. Automatic versus intentional uses of memory: Aging, attention, and control. Psychol Aging 1993;8:283-293.

61. Jonides J, Smith EE, Marshuetz C, Koeppe RA, Reuter-Lorenz PA. Inhibition in verbal working memory revealed by brain activation. Proc Nat Acad Sci USA 1998;95:8410-8413.

62. Kane MJ, Hasher L, Stoltzfus ER, Zacks RT, Connelly SL. Inhibitory attentional mechanisms and aging. Psychol Aging 1994;9:103-112.

63. Kieley JM, Hartley AA. Age-related equivalence of identity suppression in the Stroop color-word task. Psychol Aging 1997;12:22-29.

64. Klein M, Ponds R, Houx PJ, Jolles J. Effects of test duration on age-related differences in Stroop interference. J Clin Exp Neuropsychol 1997;19:77-82.

65. Koss E, Ober BA, Delis DC, Friedland RP. The Stroop Color-Word Test: indicator of dementia severity. Int J Neurosci 1984;24:53-61.

66. Kramer AF, Humphrey DG, Larish JF, Logan G., Strayer D. Aging and inhibition: Beyond an 
unitary view of inhibitory processing in attention. Psychol Aging 1994;9:491-512.

67. Langley LK, Overmier JB, Knopman DS, Prod'Homme MS. Inhibition and habituation: Preserved mechanisms of attentional selection in aging and Alzheimer's disease. Neuropsychol 1998;12:353366.

68. Langley LK, Fuentes LJ, Hochhalter AK, Brandt J, Overmier JB. Inhibition of return in aging and Alzheimer's disease: performance as a function of task demands and stimulus timing. J Clin Exp Neuropsychol 2001;23:431-446.

69. LeMoal S, Reymann JM, Thomas V, Cattenoz C, Lieury A, Allain H. Effect of normal aging and of Alzheimer's disease on episodic memory. Dementia Geriatr Cogn Dis 1997;8:281-287.

70. Light L. Memory and aging : Four hypotheses in search of data. Annual Rev Psychol 1991;42:333376.

71. Lustig CL, Hasher L, Tonev, ST. Distraction as a determinant of processing speed. Psychon Bull Rev, 2006;13:619-625.

72. MacLeod CM. Directed forgetting: The human memory literature. In : Golding JM, MacLeod CM, editors. Intentional forgetting: Interdisciplinary approaches. Mahwah, NJ: Erlbaum; 1998, p. 96103.

73. Mattis S. Dementia Rating Scale. Windsor: NFER-Nelson ; 1973.

74. May CP, Hasher L. Synchrony effects in inhibitory control over though and action. J Exp Psychol: Hum Percep Perf 1998;24:363-379.

75. McDowd JM, Oseas-Kreger DM. Aging, inhibitory processes, and negative priming. J Gerontol 1991;46:P340-P345.

76. McKhann G, Drachman D, Folstein M, Katzman R, Price D, Stadlan EM. Clinical diagnosis of Alzheimer's disease: Report of the NINCDS-ADRDA work group under the auspices of departement of health and human services task force on Alzheimer's disease. Neurology 1984;34: 939-944. 
77. Monsch AU, Bondi MW, Salmon DP, Butters N, Thal LJ, Hansen LA, Wiederholt WC, Cahn DA, Klauber MR. Clinical validity of the Mattis dementia rating scale in detecting dementia of the Alzheimer type. Arch Neurol 1995;52:899-504.

78. Moulin CJA, Perfect TJ, Conway MA, North AS, Jones RW, James N. Retrieval-induced forgetting in Alzheimer's disease. Neuropsychologia 2002;40:862-867.

79. Multhaup KS, Balota DA. Generation effects and source memory in healthy older adults and in adults with dementia of the Alzheimer type. Neuropsychol 1997;11:382-391.

80. Nielson KA, Langenecker SA, Garavan H. Differences in the functional neuroanatomy of inhibitory conrol across the adult life span. Psychol Aging 2002;17:56-71.

81. Nigg JT. On inhibition/disinhibition in developmental psychopathology: Views from cognitive and personality psychology and a working inhibition taxonomy. Psychol Bull 2000;126:220-246.

82. Park D, Hedden T. Working memory and aging. In : Naveh-Benjamin M, Moscovitch M., Roediger HLI, editors, Perspectives on human memory and cognitive aging. Essays in honour of Fergus Craick. Hove: Psychology Press; 2001, p. 148-160.

83. Paul S. Search for semantic inhibition failure during sentencecomprehension. Psychol Aging $1996 ; 11: 10-20$

84. Reed H. Studies of interference process in short-term memory. J Exp Psychol 1970;84:452-457.

85. Salmon DP. Disorders of memory in Alzheimer's disease. In: Boller F, Grafman J, editors. Handbook of Neuropsychology, 2nd Edition. Amsterdam: Elsevier Science B.V.; 2000, p. 155-195.

86. Salthouse TA, Babcock RL. Decomposing adult age differences in working memory. Develop Psychol 1991;27:763-776.

87. Salthouse TA, Becker JT. Independent effects of Alzheimer's disease on neuropsychological functioning. Neuropsychol 1998;12:242-252.

88. Salthouse TA, Meinz EJ. Aging, inhibition, working memory and speed. J Gerontol: Psychol Sci 1995;50:297-306. 
89. Salthouse TA, Thoth JP, Hancock HE, Woodard JL. Controlled and automatic forms of attention: Process purity and the uniqueness of age-related influences. J Gerontol: Psychol Sci 1997;52B: P216-P228.

90. Schneider W, Eschman A, Zuccolotto A. E-Prime user's guide. Pittsburgh, PA: Psychology Software Tools Inc; 2002.

91. Sebastian MV, Menor J, Elusoa R. Patterns of errors in short-term forgetting in AD and ageing. Memory 2001;9:223-321.

92. Shaw RJ. Age-related increases in the effects of automatic semantic activation. Psychol Aging 1991;6:595-604.

93. Shilling VM, Chetwynd A, Rabbitt PMA. Individual inconsistency across measures of inhibition: an investigation of the construct validity of inhibition in older adults. Neuropsychologia 2002;40: 605-619.

94. Spieler DH, Balota DA, Faust ME. Stroop performance in healthy younger and older adults and in individuals with dementia of the Alzheimer's type. J Exp Psychol 1996;22:461-479.

95. Stoltzfus ER, Hasher L, Zacks RT, Ulivi MS, Goldstein D. Investigation of inhibition and interference in younger and older adults. J Gerontol 1993;48:P179-P188.

96. Sullivan MP, Faust ME, Balota DA. Identity negative priming in older adults and individuals with dementia of the Alzheimer type. Neuropsychol 1995;9:537-555.

97. Teresa Lechuga $\mathrm{M}^{\mathrm{a}}$, Moreno V, Pelegrina S, Gomez-Ariza CJ, Bajo Teresa $\mathrm{M}^{\mathrm{a}}$. Age differences in memory control: Evidence from updating and retrieval practice tasks. Acta Psychologica 2006;123:279-298.

98. Tipper SP. Less attentional selectivity as a result of declining inhibition in older adults. Bull Psychonomic Soc 1991;29:45-47.

99. Titov N, Knight RG. Adult age differences in controlled and automatic memory processing. Psychol Aging 1997;12:565-573. 
100. Van der Linden M, Hupet M, Feyereisen P, Schelstraete M, Bestgen Y, Bruyer R, Lories G, El Ahmadi A, Seron X. Cognitive mediators of age-related differences in langage comprehension and verbal memory performance. Aging Neuropsychol Cogn 1999;6:32-55.

101. Verhaeghen P, De Meersman L. Aging and the Stroop effect: A meta-analysis. Psychol Aging 1998;13:120-126.

102. Verhaeghen P, De Meersman L. Aging and the negative priming effect: a meta-analysis. Psychol Aging 1998;13:435-444.

103. Weingartner H, Kaye W, Smallberg SA, Ebert MH, Gillin JC, Sitaram N. Memory failures in progressive idiopathic dementia. J Abnormal Psychol 1981;90:187-196.

104. Wilson SP, Kipp K. The development of efficient inhibition: Evidence from directed forgetting tasks. Develop Rev 1998;18:86-123.

105. Yoon C, May CP, Hasher L. Aging, circadian arousal patterns, and cognition. In : Park D., Schwartz N., editors. Cognitive aging: A primer. Hove: Psychology Press; 2000, p. 151-170.

106. Zacks RT, Hasher L, Radvansky G. Studies of directed forgetting in older adults. J Exp Psychol: Learning, Mem Cogn 1996;22:143-156. 
Table 1. Probe recency task. Response time (in ms; mean \pm standard deviation) and number of correct responses [out of 20; mean (standard deviation)] as a function of group and condition.

\begin{tabular}{|l|c|c|c|}
\hline & Young subjects & Elderly subjects & Alzheimer patients \\
\hline Response times & $895(269)$ & $1,376(255)$ & $2,808(1154)$ \\
Ronrecent Negative & $953(280)$ & $1,435(270)$ & $2,910(1373)$ \\
Nonrecent Positive & $930(277)$ & $1,367(304)$ & $2,487(1267)$ \\
Recent Positive & $917(251)$ & $1,369(304)$ & $2,319(1151)$ \\
Interference effect & $0.064(0.08)$ & $0.041(0.10)$ & $0.023(0.20)$ \\
Facilitation effect & $-0.008(0.09)$ & $0.002(0.12)$ & $-0.072(0.12)$ \\
& & & \\
Correct responses & $19.43(0.90)$ & $19(1.32)$ & $16.65(3.1)$ \\
Nonrecent Negative & $19.33(0.76)$ & $18.30(1.52)$ & $15.60(2.76)$ \\
Recent Negative & $18.60(1.65)$ & $17.75(1.86)$ & $15.25(3.18)$ \\
Nonrecent Positive & $18.87(1.33)$ & $18.45(1.15)$ & $16.5(2.91)$ \\
Recent Positive & $-0.005(0.06)$ & $-0.041(0.08)$ & $-0.059(0.20)$ \\
Interference effect & $0.016(0.08)$ & $0.042(0.12)$ & $0.086(0.15)$ \\
Facilitation effect & & & \\
\hline
\end{tabular}


Table 2. Flanker task. Response time [in ms; mean (standard deviation)] and number of correct responses (out of 32) as a function of group and condition

\begin{tabular}{|l|c|c|c|}
\hline & Young subjects & Elderly subjects & Alzheimer patients \\
\hline Response times & $703(131)$ & $906(290)$ & $1,324(628)$ \\
Interference condition & $740(134)$ & $900(267)$ & $1,484(974)$ \\
Neutral condition & $725(126)$ & $904(290)$ & $1,540(1086)$ \\
Interference effect & $-0.020(0.05)$ & $0.004(0.06)$ & $0.015(0.086)$ \\
Facilitation effect & $0.032(0.04)$ & $-0.003(0.04)$ & $0.07(0.18)$ \\
Correct responses & $31.35(0.72)$ & $31.30(1.17)$ & $31.27(1.31)$ \\
Facilitation condition & $31.27(1.05)$ & $31.45(1)$ & $29.05(4.63)$ \\
Interference condition & $31.13(1.11)$ & $30.70(0.73)$ & $29.75(2.07)$ \\
Neutral condition & $-0.004(0.04)$ & $-0.024(0.023)$ & $0.040(0.15)$ \\
Interference effect & $-0.007(0.03)$ & $-0.019(0.02)$ & $-0.05(0.07)$ \\
Facilitation effect & & & \\
& & & \\
\hline
\end{tabular}


Table 3. Directed forgetting task. Number of correct responses and errors [mean (standard deviation)] as a function of group and condition.

\begin{tabular}{|l|c|c|c|}
\hline & Young subjects & Elderly subjects & Alzheimer patients \\
\hline Control condition & $29.30(0.88)$ & $29.75(0.71)$ & $29(1.27)$ \\
Retroactive interference condition & $23.37(3.31)$ & $25.95(4.11)$ & $18.40(5.17)$ \\
Inhibition condition & $24.53(3.60)$ & $24.80(3.90)$ & $18.20(6.04)$ \\
Directed forgetting cost & $1.53(3.46)$ & $-1.15(3.10)$ & $-0.20(4.31)$ \\
Omission errors (all conditions) & $0(0)$ & $2(2.4)$ & $5.8(6.8)$ \\
Position errors (all conditions) & $0.2(1.1)$ & $2.10(2.02)$ & $2.95(1.93)$ \\
Intrusion errors TBF & $3.17(2.71)$ & $3.65(3.09)$ & $8.95(6.52)$ \\
\hline
\end{tabular}


Table 4. Hayling task. Response time [in sec.; mean (standard deviation)] and accuracy of response [mean (standard deviation)] as a function of group and part of the task.

\begin{tabular}{|l|c|c|c|}
\hline & Young subjects & Elderly subjects & Alzheimer patients \\
\hline Response time (Part A) & $1.03(0.07)$ & $1.39(0.93)$ & $1.59(0.77)$ \\
Errors (Part A) & $0.03(0.18)$ & $0.50(1.47)$ & $0.85(1.23)$ \\
Response time (Part B - Part A) & $1.63(1.17)$ & $4.24(2.23)$ & $5.61(4.79)$ \\
Semantic relatedness (Part B) & $4.33(2.56)$ & $8.05(3.63)$ & $14.15(0.38)$ \\
\hline
\end{tabular}


Table 5. Summary of the inhibitory effects observed in elderly subjects and AD patients for the different tasks.

\begin{tabular}{|c|c|c|}
\hline Task & Elderly subjects & AD patients \\
\hline \multicolumn{3}{|l|}{ Probe recency } \\
\hline Response time & Preserved & Preserved \\
\hline Accuracy of response & Preserved & Preserved \\
\hline \multicolumn{3}{|l|}{ Directed forgetting } \\
\hline Recall of TBR consonants & Impaired & No more impaired \\
\hline TBF intrusions errors & Preserved & Impaired \\
\hline \multicolumn{3}{|l|}{ Flanker } \\
\hline Response time & Preserved & Preserved \\
\hline Accuracy of response & Preserved & Impaired \\
\hline \multicolumn{3}{|l|}{ Hayling } \\
\hline Response time & Impaired & No more impaired \\
\hline Semantic relatedness & Impaired & Impaired* \\
\hline
\end{tabular}

* explained by span size and processing speed 